\title{
A Network-Based Enhanced Spectral Diversity Approach for TOPS Time-Series Analysis
}

\author{
Heresh Fattahi, Member, IEEE, Piyush Agram, Member, IEEE, and Mark Simons
}

\begin{abstract}
For multitemporal analysis of synthetic aperture radar (SAR) images acquired with a terrain observation by progressive scan (TOPS) mode, all acquisitions from a given satellite track must be coregistered to a reference coordinate system with accuracies better than 0.001 of a pixel (assuming full SAR resolution) in the azimuth direction. Such a high accuracy can be achieved through geometric coregistration, using precise satellite orbits and a digital elevation model, followed by a refinement step using a time-series analysis of coregistration errors. These errors represent the misregistration between all TOPS acquisitions relative to the reference coordinate system. We develop a workflow to estimate the time series of azimuth misregistration using a network-based enhanced spectral diversity (NESD) approach, in order to reduce the impact of temporal decorrelation on coregistration. Example time series of misregistration inferred for five tracks of Sentinel-1 TOPS acquisitions indicates a maximum relative azimuth misregistration of less than 0.01 of the full azimuth resolution between the TOPS acquisitions in the studied areas. Standard deviation of the estimated misregistration time series for different stacks varies from 1.1e-3 to $2 \mathrm{e}-3$ of the azimuth resolution, equivalent to $1.6-2.8 \mathrm{~cm}$ orbital uncertainty in the azimuth direction. These values fall within the 1-sigma orbital uncertainty of the Sentinel-1 orbits and imply that orbital uncertainty is most likely the main source of the constant azimuth misregistration between different TOPS acquisitions. We propagate the uncertainty of individual misregistration estimated with ESD to the misregistration time series estimated with NESD and investigate the different challenges for operationalizing NESD.
\end{abstract}

Index Terms-Coregistration, interferometric synthetic aperture radar (InSAR), spectral diversity, terrain observation by progressive scan (TOPS).

\section{INTRODUCTION}

$\mathbf{T}$ ERRAIN Observation by Progressive Scan (TOPS) [1] is the default mode of operation for the Sentinel-1 synthetic aperture radar (SAR) satellites [2]. The use of data acquired in TOPS mode for interferometric SAR (InSAR) has been first demonstrated using observations from the TerraSAR-X satellite [3]-[5]. With the launch of Sentinel-1A, TOPS has been used to image the ground surface displacements caused by several earthquakes, including 2015 Mw 7.8 Gorkha, Nepal [6], 2016 Mw 7.2 Kumamoto, Japan [7], $2015 \mathrm{Mw} 8.3$ Illapel, Chile [8], and $2015 \mathrm{Mw}$

Manuscript received July 25, 2016; revised September 9, 2016 and September 22, 2016; accepted September 30, 2016. The work of M. Simons was supported by the National Aeronautics and Space Administration under Grant NNX16AK58G.

H. Fattahi and M. Simons are with the California Institute of Technology, Pasadena, CA 91125 USA (e-mail: hfattahi@gps.caltech.edu).

P. Agram is with the Jet Propulsion Laboratory, California Institute of Technology, Pasadena, CA 91109 USA.

Color versions of one or more of the figures in this paper are available online at http://ieeexplore.ieee.org.

Digital Object Identifier 10.1109/TGRS.2016.2614925
7.2 Tajikistan [9]. Sentinel-1 TOPS data have also been used to image the ground deformation due to the 2014-2015 volcanic eruptions at the Pico de Fogo volcano [10] and to map the surface velocity of ice sheets and glaciers in Greenland [11].

InSAR processing requires precise alignment and registration of two complex images. Techniques for achieving the necessary coregistration accuracy include coherent or incoherent cross correlation and approaches based on imaging geometry. Their coregistration accuracy of approximately 0.01 of an SAR resolution cell across the satellite track (i.e., the range direction) and along the satellite track (i.e., the azimuth direction) is adequate for traditional InSAR using stripmap acquisitions [12]-[15]. In contrast, as we discuss further in Section III, TOPS InSAR requires an unprecedented coregistration accuracy an order of magnitude finer, i.e., better than 0.001 of an azimuth resolution cell in order to avoid phase discontinuities in the resulting interferograms [4].

Precise offsets between TOPS images can be obtained by geometry-based coregistration refined by finer azimuth misregistration [4], [16], [17]. The misregistration between two coregistered SAR images can be estimated using cross correlation of the two images or using the spectral diversity approach [16], [18]. In the latter approach, the difference in the spectrum (spectral separation) of subband SAR images is used to estimate the misregistration. The subband SAR images can be obtained by focusing the SAR data to two slightly different frequencies in range or azimuth directions or by splitting the spectrum of a full-band SAR image to two subband images using a bandpass filter. Differential interferometric phase of the sublook images divided by their frequency separation results in the misregistration between the two SAR images [19]. The coregistration accuracy of the offsets derived from spectral diversity is comparable with the cross-correlation techniques [14].

Once processing schemes for SAR image focusing accurately account for acquisition geometry [20], sources of misregistration between SAR images reduce to errors in the satellite state vector (orbital error), timing errors in the SAR instrument (timing error), error in the digital elevation model (DEM) used for the geometric coregistration (DEM error), and propagation delay through troposphere and ionosphere [17], [21]. These remaining errors may cause constant or spatially variable azimuth misregistration across SAR images.

In the case of a constant azimuth misregistration, an enhanced spectral diversity (ESD) approach can be used to estimate the azimuth misregistration with accuracies bet- 
ter than 0.001 SAR azimuth cell, significantly higher than the accuracy of the spectral diversity and cross-correlation techniques [4], [16]-[18]. The performance of ESD has been evaluated in different studies using TOPS data acquired by TerraSAR-X [4], [5], [16] and Sentinel-1A satellites [21].

The accuracy of the azimuth misregistration estimated with ESD, is controlled by the coherence between SAR images. Therefore, temporal decorrelation can significantly affect the accuracy of the azimuth misregistration estimated by the ESD approach [4]. Temporal decorrelation is of particular concern when a stack of TOPS images should be coregistered to a master coordinate system for the multitemporal analysis of SAR data, such as needed for InSAR time-series analysis. Here, a stack refers to an ensemble of TOPS images acquired from the same satellite track over the same region at different acquisition times. Increasing the number of acquisitions in a given stack will increase the sensitivity of coregistration to temporal decorrelation. To reduce the impact of temporal decorrelation, we develop a network-based ESD (NESD) approach in which we form a network of short temporal baseline pairs of TOPS images to estimate the azimuth misregistration of all images with respect to a reference coordinate system. The performance of a similar independently developed approach has been recently presented and compared with single-master coregistration assuming a temporal decay of the coherence [22]. Here, we evaluate the performance of NESD in more detail by investigating the uncertainty of the estimated misregistration time series obtained with NESD compared with differential misregsitration obtained with ESD. We discuss the possible limitations for operationalizing NESD, including large and nonstationary azimuth misregistration, large ground displacement in the azimuth direction, and abrupt coherence loss, which violates the assumption of temporal decay of coherence. We also evaluate the performance of NESD and the temporal variation of the misregsitration using several stacks of Sentinel-1 data in different regions of the world.

We organize this paper as follows. Section II reviews the TOPS imaging geometry and its spectral characteristics in the azimuth direction. Section III discusses the impact of azimuth misregistration on the interferometric phase. Section IV evaluates the theoretical accuracy of ESD for estimating the azimuth misregistration. Section V formulates the NESD approach to estimate the misregistration time series, propagates the uncertainty of individual misregistration from ESD to misregistration time series from NESD, and evaluates the theoretical accuracy of NESD. Section VI presents a workflow for coregistering a stack of TOPS images and Section VII shows experimental results obtained with the proposed workflow. Section VIII discusses several limitations for operationalizing NESD.

\section{TOPS GEOMETRY}

In conventional stripmap SAR imaging, the radar antenna is fixed to a specific direction illuminating a single swath of the scene with a fixed squint angle (i.e., the angle between the radar beam and the range direction) (Fig. 1). The imaging swath width can be increased using the scanning SAR

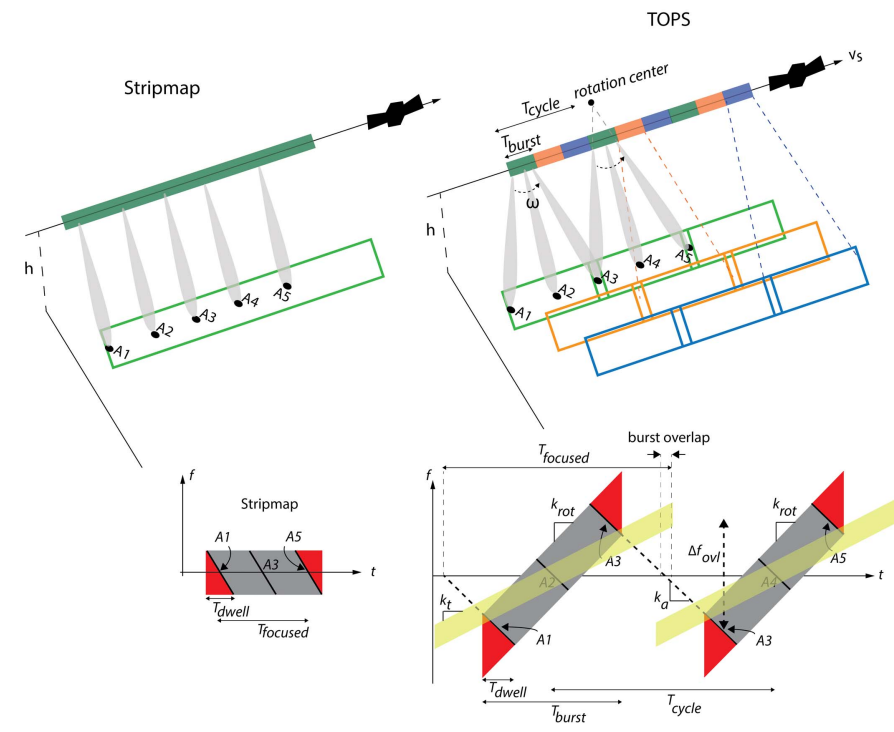

Fig. 1. Imaging geometry and time-frequency diagram for conventional stripmap and TOPS. (Top left) Stripmap acquisition geometry. (Top right) TOPS acquisition geometry. (Bottom left) Time-frequency diagram for stripmap. (Bottom right) Time-frequency diagram for two consecutive burst raw data (gray) and focused burst SLC (yellow). The red regions in the burst raw data diagram show data that is not useful for this application. A1-A5 are five targets on the ground illuminated by stripmap and TOPS. Solid lines in the time-frequency diagrams show the Doppler history of the targets. $K_{\text {rot }}$ denotes the Doppler centroid rate caused by the azimuth beam steering, $K_{a}$ is the Doppler rate of the target, and $K_{t}$ denotes the Doppler rate for the focused burst image.

(ScanSAR) burst mode imaging technique in which the elevation beam (radar beam in range direction) is switched between different subswaths in the range direction between the successive bursts [23], [24]. In the ScanSAR acquisition mode, similar to stripmap mode, the radar beam is kept at constant squint angle. The larger swath coverage of ScanSAR mode compared with stripmap mode comes at the expense of degraded azimuthal resolution. In practice, switching the elevation beam in the range direction, enforces a shorter dwell time (i.e., the time that a target is illuminated) at each subswath, limits the Doppler history, reduces the target bandwidth, and degrades the azimuth resolution [25], [26]. An important characteristic of ScanSAR acquisitions is the spatially varying spectrum of the data, which leads to a periodic modulation of the amplitude, or scalloping, and azimuth varying resolution, ambiguity ratio, and signal-to-noise ratio (SNR) [25], [26].

TOPS achieves the wide swath coverage without usual ScanSAR problems by electrical steering of the azimuth beam from aft to the fore with a constant rotation rate [1]. While an azimuth varying azimuth antenna pattern (AAP) in the ScanSAR causes scalloping and azimuth varying resolution, azimuth beam steering in the TOPS acquisitions ensures the same AAP for every acquired target, leading to uniform azimuth resolution and SNR in the azimuth direction without scalloping in the data [1].

As with the ScanSAR mode, the wide swath of the TOPS acquisitions is obtained by degrading the azimuth resolution compared with the conventional stripmap mode. However, the mechanism controlling the reduction of the azimuth resolution with TOPS is different from ScanSAR. ScanSAR decreases 
azimuth resolution by slicing the footprint of the azimuth beam due to switching the elevation beam to cover wider swaths in range [1]. With TOPS, the footprint of the radar is shrunk by a factor of $\alpha$ compared with the fixed azimuth beam in the stripmap [1]. We can write

$$
\alpha=1+\frac{r_{0}|\omega|}{v_{s}}
$$

where $r_{0}$ is the closest range to the target, $\omega$ denotes the steering rate of the azimuth beam, and $v_{s}$ represents the satellite velocity. The azimuth resolution for fixed stripmap radar with length of $L$ is given by $\delta_{\text {stripmap }}=L / 2$ and degrades by a factor of $\alpha$ for TOPS images as $\delta_{\text {TOPS }}=\alpha \delta_{\text {stripmap }}$. Assuming $\omega=1.5^{\circ} / \mathrm{s}, r_{0}=830 \mathrm{~km}$, and $v_{s}=7200 \mathrm{~m} / \mathrm{s}$ (values chosen to roughly correspond to Sentinel-1), equation (1) gives $\alpha \approx 4$.

Fig. 1 shows the stripmap and TOPS acquisition geometries together with their corresponding time-frequency diagrams. For stripmap, the Doppler centroid frequency is constant with time. The Doppler rate of the target is given by [1], [4]

$$
K_{a}=-\frac{2 v_{\text {eff }}^{2}}{\lambda r}
$$

where $v_{\text {eff }}$ is the effective velocity of the target and depends on the satellite ephemerides and target position. $v_{\text {eff }}$ relates to ground velocity $\left(v_{g}\right)$ and satellite velocity $\left(v_{s}\right)$ as $v_{\text {eff }}^{2}=v_{g} v_{s}$.

In the TOPS geometry, in addition to the Doppler rate of the target, the counterclockwise rotation of the azimuth beam, with a steering rate of $\omega$, introduces a linear variation to the Doppler centroid within each burst with a rate of $K_{\text {rot }}$ given by [1], [4]

$$
K_{\text {rot }}=-\frac{2 v_{\text {eff }}^{2}}{\lambda r_{\text {rot }}} \approx \frac{2 v_{s}}{\lambda} \omega
$$

where $r_{\text {rot }}$ is the distance from the virtual rotation center of the acquisition to the target. After focusing the burst raw data to the burst single-look complex (SLC) in zero-Doppler geometry, the Doppler centroid of the burst images varies as a function of time with a rate of $K_{t}$. Consideration of the imaging geometry gives [4]

$$
K_{t}=\frac{K_{a} K_{\mathrm{rot}}}{K_{a}-K_{\mathrm{rot}}} .
$$

The linear variation of the Doppler frequency within each burst SLC, results in a spectral separation $\Delta f_{\text {ovl }}$ between two consecutive burst images at their overlap region [Fig. 1(d)]. The spectral separation equals to the magnitude of the frequency variation within a burst and can be obtained as $\Delta f_{\text {ovl }}=K_{t} T_{\text {focused }}$. This spectral separation is used for estimating the azimuth misregistration in Section IV.

\section{Interferometric Phase Due to COREGISTRATION ERROR}

We begin with two SAR images, here referred to as the master and the slave, focused to the same Doppler centroid frequency $f_{c}$, and a common bandwidth $B$, where the slave image is coregistered and resampled to the master image. The

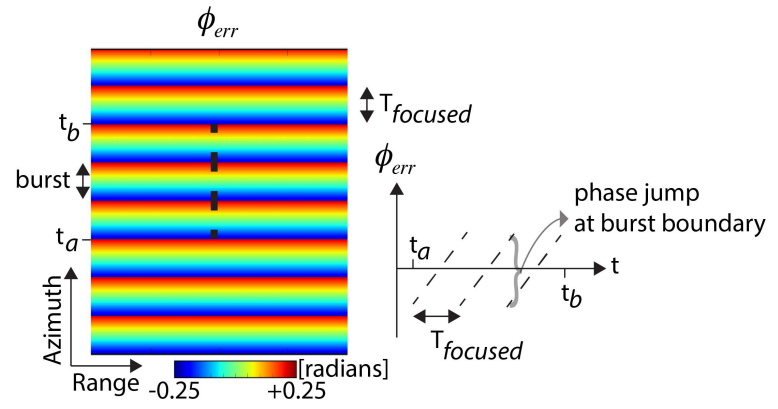

Fig. 2. (Left) Expected azimuth phase ramps in Sentinel-1 TOPS interferograms due to a constant azimuth misregistration of 0.003 azimuth cell. (Right) Transect in the azimuth direction showing the phase discontinuity between consecutive bursts caused by the constant azimuth misregistration.

impulse response functions of a target in the two images as a function of azimuth time is given as [19]

$$
\begin{aligned}
M(t) & =e^{j \phi_{M}} e^{j 2 \pi f_{c}\left(t-t_{0}\right)} \operatorname{sinc}\left(\pi B\left(t-t_{0}\right)\right) \\
S(t) & =e^{j \phi_{S}} e^{j 2 \pi f_{c}\left(t-t_{0}-\Delta t\right)} \operatorname{sinc}\left(\pi B\left(t-t_{0}-\Delta t\right)\right)
\end{aligned}
$$

where $\phi_{M}$ and $\phi_{S}$ are the signal phase at the master and slave images, $t_{0}$ is the zero frequency position of the target, and $\Delta t$ is the azimuth misregistration of the slave image relative to the master image. The phase difference between the two signals equals $\phi_{M S}(t)=\phi_{M}(t)-\phi_{S}(t)+\phi_{\mathrm{err}}(t)$, where the interferometric phase due to the range change from the radar to the target is $\left(\phi_{M}-\phi_{S}\right)=\frac{4 \pi}{\lambda}\left(r_{M}-r_{S}\right)$, with $r_{M}$ and $r_{S}$ denoting the range to the same target in the master and slave acquisitions, respectively, and $\phi_{\text {err }}$ represents the phase due to an azimuth misregistration of $\Delta t$ as [19]

$$
\phi_{\mathrm{err}}(t)=2 \pi f_{c}(t) \Delta t
$$

Since $\phi_{\text {err }}$ is frequency dependent, a constant azimuth misregistration between two stripmap images (with constant Doppler centroid frequency in azimuth) results in a constant phase error. In TOPS images, the Doppler frequency varies linearly with time (Fig. 1). Therefore, a constant azimuth misregistration leads to a phase ramp in the azimuth direction in a TOPS burst interferogram. Due to the cyclic variation of the Doppler centroid frequency of the consecutive focused bursts (Fig. 1), the difference between two phase ramps at the overlap of the consecutive burst interferograms, appears as a phase discontinuity at the burst overlaps in the TOPS interferograms (Fig. 2).

\section{Enhanced Spectral Diversity}

Given two subsequent burst images at a master date $\left(M_{i}\right.$ and $\left.M_{i+1}\right)$ and their corresponding slave burst images $\left(S_{i}\right.$ and $S_{i+1}$ ), a differential interferogram can be formed at the overlap region of the bursts as [4]

$$
\phi_{\mathrm{ovl}}=\phi_{i}-\phi_{i+1}=\arg \left(M_{i} . S_{i}^{*}\right)-\arg \left(M_{i+1} . S_{i+1}^{*}\right)
$$

where $\phi_{\text {ovl }}$ is the phase of the differential overlap interferogram. In the absence of significant ground displacement in the azimuth direction and assuming negligible residual geometric phase, $\phi_{\mathrm{ovl}}$ represents the phase due to the azimuth 


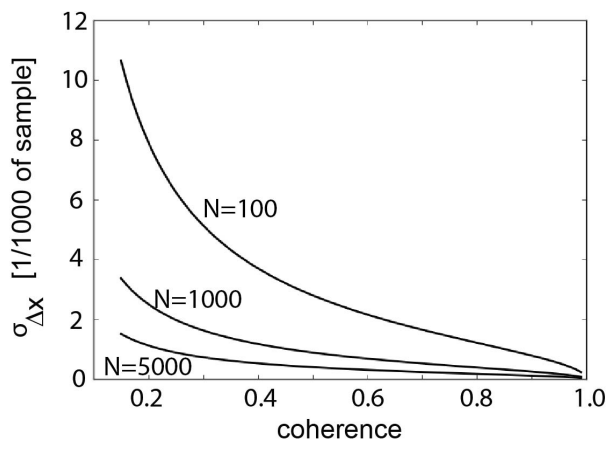

Fig. 3. Theoretical accuracy of the estimated misregistration using ESD as a function of coherence for different numbers of samples assuming $\Delta f_{\text {ovl }}=$ $4790 \mathrm{~Hz}$ and $\tau=0.002056 \mathrm{~s}$. High coherence and larger number of samples improve the accuracy of the estimated misregistration.

misregistration. From (7) and (8), the azimuth misregistration is obtained as

$$
\Delta t=\frac{\phi_{\text {ovl }}}{2 \pi \Delta f_{\text {ovl }}}
$$

where $\Delta f_{\text {ovl }}$ can be computed from (4). The azimuth misregistration in terms of the image samples in the azimuth direction is obtained as $\Delta x=\Delta t / \tau$, where $\tau$ is the azimuth time interval (image sampling in the azimuth direction) in seconds. Constant azimuth misregistration may be due to the orbital error in the azimuth direction, timing error of the SAR instrument, or spatially constant ground displacement (e.g., solid earth tide).

The accuracy of the estimated misregistration is given as

$$
\sigma_{\Delta x}=\frac{1}{2 \pi \Delta f_{\text {ovl }} \tau} \sqrt{\sigma_{\phi_{i}}^{2}+\sigma_{\phi_{i+1}}^{2}-2 \sigma_{\phi_{i}, \phi_{i+1}}} .
$$

Ignoring the covariance between the two interferograms at the overlap region of two consequtive bursts $\left(\sigma_{\phi_{i}, \phi_{i+1}}=0\right)$, and assuming similar phase variance for the two interferograms, (10) simplifies to

$$
\sigma_{\Delta x}=\frac{\sqrt{2} \sigma_{\phi}}{2 \pi \Delta f_{\mathrm{ovl}} \tau}
$$

where $\sigma_{\phi}$, the phase noise standard deviation, can be approximated as

$$
\sigma_{\phi}=\frac{1}{\sqrt{2 N}} \frac{\sqrt{1-\gamma^{2}}}{\gamma}
$$

where $N$ is the number of independent averaged samples and $\gamma$ is the interferometric coherence. Fig. 3 shows the accuracy of the estimated misregistration with ESD as a function of interferometric coherence given different numbers of samples.

\section{Network-Based Enhanced Spectral Diversity}

Multitemporal analysis of TOPS SAR data, such as InSAR time-series analysis, requires the coregistration of TOPS images to a common master coordinate system, hereafter called the stack master. When temporal decorrelation is proportional to the time difference between the two SAR images (i.e., when coherence decreases with time), the accuracy of the misregistration estimated by ESD decreases with increasing time difference between the stack master and the slave

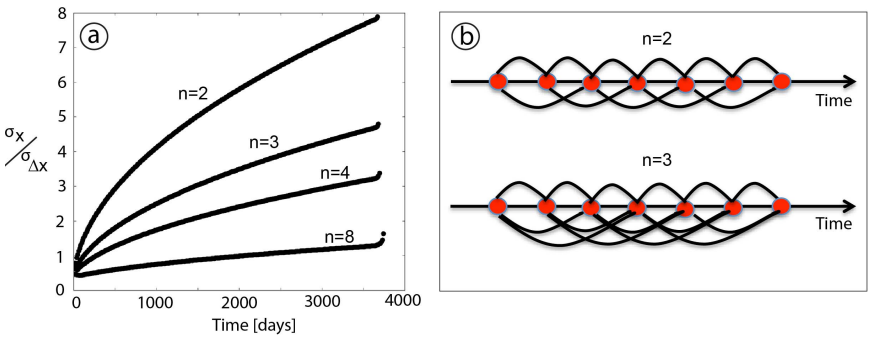

Fig. 4. (a) Ratio of the time-series misregistration accuracy obtained with $\operatorname{NESD}\left(\sigma_{x}\right)$ and the individual pair misregistration obtained with $\operatorname{ESD}\left(\sigma_{\Delta x}\right)$ as a function of time, for different sequential networks of small temporal baseline interferograms in which each acquisition is connected to $n$ following acquisition. Ten years of acquisitions with acquisition interval of 12 days was assumed. (b) Examples of the sequential network of interferograms, with circles showing the SAR acquisitions and arcs representing the interferograms.

images. To overcome this problem, we use a network of small temporal baseline overlap interferograms to estimate the differential misregistration between coherent pairs, and then invert the differential misregistration to estimate the time series of misregistration for the TOPS images.

The differential misregistration relates to the time series of the misregistration using a linear system of equations as $\Delta \mathbf{x}=\mathbf{A} \mathbf{x}$, where $\Delta \mathbf{x}$ is a vector of differential azimuth misregistration estimated with ESD for each pair, $\mathbf{A}$ is a design matrix (also referred to as the incidence matrix in graph theory terminology), and $\mathbf{x}$ is the vector of misregistration time series for the stack of TOPS images relative to the stack-master acquisition. $\mathbf{x}$ can be inferred using a simple least-squares or weighted least-squares estimation approach. To ensure the unbiased estimation of $\mathbf{x}$, we use a connected network of overlap interferograms. Note that estimating the misregistration time series from the differential misregistration of small-baseline pairs in NESD is similar to estimating the displacement time series from the differential displacements [33], [36], [37].

Given a full covariance matrix for the vector of differential azimuth misregistration $\mathbf{C}_{\boldsymbol{\Delta} \mathbf{x}}$, the covariance matrix of the misregistration time series is obtained as $\mathbf{C}_{\mathbf{x}}=\left(\mathbf{A}^{T} \mathbf{C}_{\mathbf{A x}}^{-1} \mathbf{A}\right)^{-1}$. Assuming the same uncertainty of $\sigma_{\Delta x}$ for all differential misregistration and ignoring the covariance between elements of $\Delta \mathbf{x}$, the covariance matrix of estimated misregistration time series is given by $\mathbf{C}_{\mathbf{x}}=\sigma_{\Delta x}^{2}\left(\mathbf{A}^{T} \mathbf{A}\right)^{-1}$. Diagonal elements of $\mathbf{C}_{\mathbf{x}}$ represent the variance of estimated misregistration for each image relative to the stack master denoted by $\sigma_{x}^{2}$. It is worth noting that if a temporal model is assumed for the misregistration time series, the variance of the model coefficients can be easily obtained using an analytical formula similar to what has been previously done for the rate of displacement time series in [36].

Fig. 4(a) shows $\sigma_{x} / \sigma_{\Delta x}$ as a function of time for a stack of TOPS acquisitions with 12 days acquisition interval over 10 years, for different sequential networks of small temporal baseline interferograms, in which each acquisition is connected to $n$ following acquisitions (see Fig. 4(b) for examples of the sequential networks). Fig. 4(a) shows that the uncertainty of the misregistration increases with time difference from the reference acquisition (in this case the first acquisition). For example, for a network with $n=4$, the uncertainty of the 


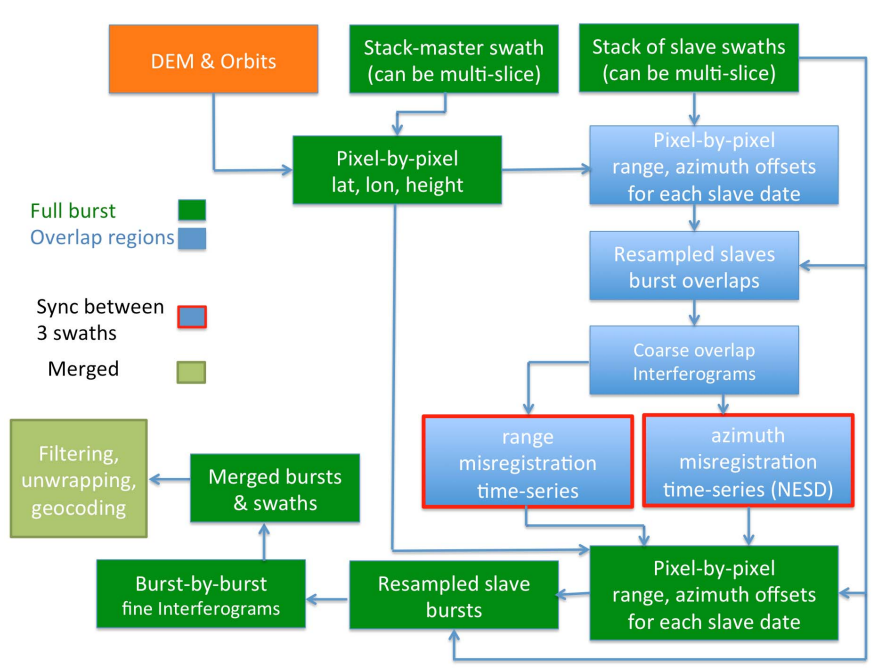

Fig. 5. InSAR processing workflow for Sentinel-1 TOPS processing based on NESD.

NESD misregistration after 5.5 and 10 years is double and triple of the ESD uncertainty, respectively. In theory, assuming an average coherence of 0.75 , ESD accuracy of 0.0002 of azimuth cell is achievable by averaging the ESD misregistration from 5000 pixels. Accordingly, NESD accuracies of 0.0004 and 0.0006 of an azimuth resolution cell over 5.5 and 10 years are achievable.

Note that Fig. 4(a) was obtained assuming the same accuracy for ESD misregistration from different interferograms, ignoring the temporal decorrelation of longer temporal baseline neighboring interferograms. This assumption can be violated for large values of $n$. The value of $n$ for which the assumption is violated, varies for different regions based on local scattering properties. In this paper, we use $n \leq 4$.

\section{InSAR PRocessing WORKFlow FOR SENTINEL-1 TOPS DATA BASED ON NESD}

Fig. 5 summarizes the burstwise workflow to coregister a stack of TOPS acquisitions. In this workflow, the burst overlaps of all slave acquisitions are coregistered to the stack master using precise orbits and a DEM. We then form a network of small temporal baseline burst overlap interferograms and estimate the azimuth misregistration of all slave acquisitions with respect to the stack master using the NESD approach. Misregistration time series in the range direction can also be estimated from a network of individual pairs of range misregistration similar to NESD. The range misregistration for individual pairs cannot be estimated with ESD; they can be estimated using cross-correlation techniques or using spectral diversity in the range direction. At the next step, we precisely coregister the full burst SLCs to the stack master using the geometrical range and azimuth offsets and the range and azimuth misregistration time series.

Note that we only use a connected network of small temporal baseline interferograms for reliably estimating the azimuth misregistration. Once NESD has been applied, any interferometric network can be generated for deformation time-series analysis from amongst these set of coregistered SLC images by simple cross multiplication at the burst level.

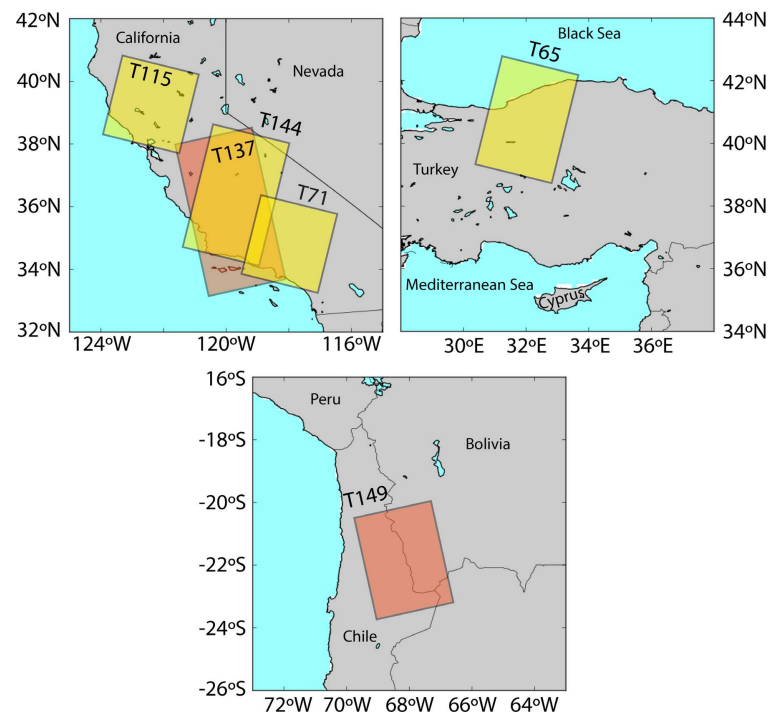

Fig. 6. Footprints of Sentinel-1A TOPS acquisitions in different active tectonic regions.

In slowly deforming areas, full swath interferograms are obtained by merging the burst interferograms and the single swath interferograms. Afterward, multilooking, filtering, and phase unwrapping can be applied on the merged interferograms as commonly done with traditional InSAR processing.

\section{EXPERIMENTAL RESUlTS}

We evaluate the azimuth misregistration in six different stacks of TOPS images acquired by the Sentinel-1A over various tectonic regions in west and south western U.S., Turkey, and Northern Chile (Fig. 6). We investigate the impact of the precise coregistration using NESD on the TOPS interferograms by visually evaluating the phase discontinuities at burst boundaries before and after applying the azimuth misregistration. We also quantify the temporal variation of the estimated azimuth misregistration.

Fig. 7 shows zoomed-in view of two interferograms from tracks 115 in California and track 64 over Turkey, both with and without applying the constant azimuth misregistration estimated from NESD. Fig. 7 shows that correcting for constant azimuth misregistration removes the phase discontinuities at the burst boundaries of these two interferograms.

Visual investigation of small-baseline interferograms from five tracks traversing western U.S. and Turkey formed from 100 TOPS images acquired by Sentinel-1A indicates no phase discontinuity in any of the interferograms after correcting the azimuth misregistration obtained for each track using the NESD approach. For Track 149 over Northern Chile, we identify a few interferograms with phase discontinuities at the overlap regions, caused by spatially variable azimuth misregistration, most likely due to the impact of strong temporal variations in the ionosphere, which we discuss in more detail in Section VIII.

\section{A. Temporal Variation of the Azimuth Misregistration}

Fig. 8 shows the time series of the constant azimuth misregistration for five different tracks, which NESD removes 

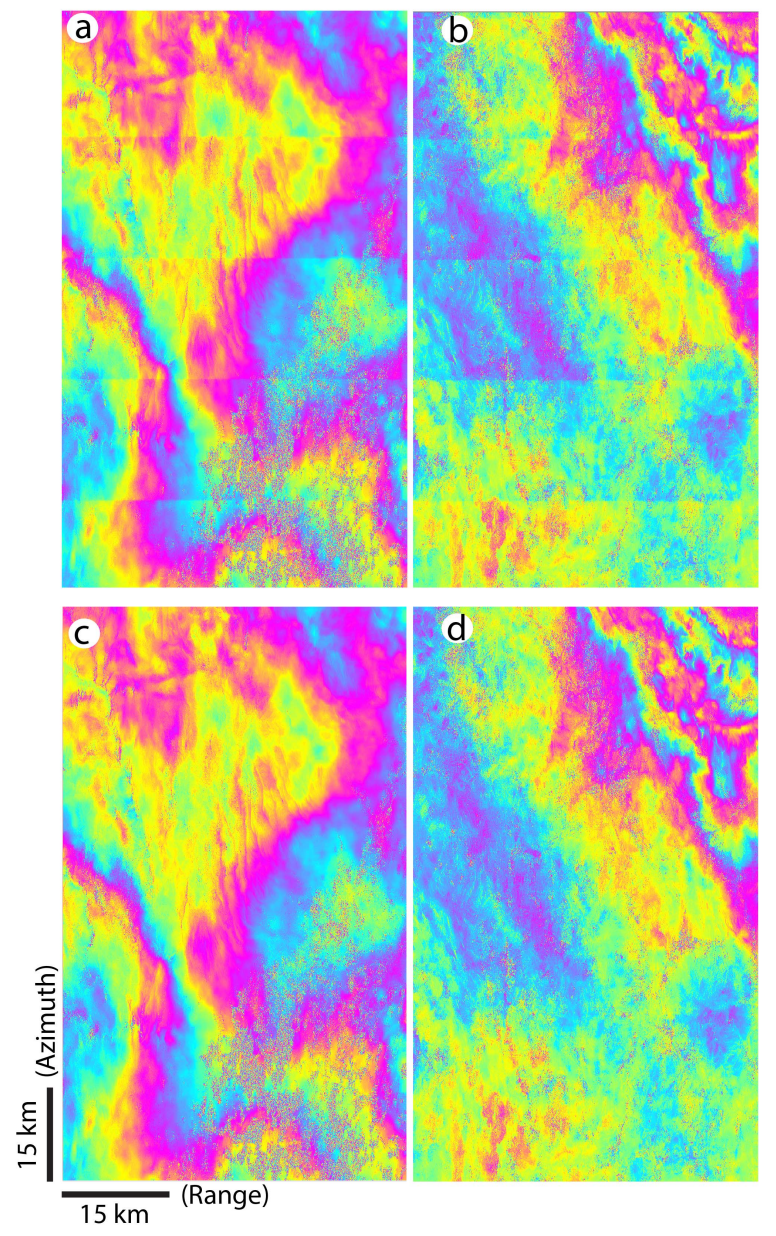

Fig. 7. Examples of Sentinel-1 TOPS interferograms before and after correcting the azimuth misregistration using NESD. A zoomed-in view to an interferogram from (a) descending track 115 between acquisitions 20150821 and 20150914 before NESD, (b) track 64 across the North Anatolian Fault in Turkey between acquisitions 20150701 and 20150713 before NESD, (c) same as (a) but after NESD, and (d) same as (b) but after NESD.

all phase discontinuities at burst overlaps over entire stack. For each stack, the misregistration is relative to the first acquisition. The relative constant misregistration does not exceed 0.01 azimuth cell. Note that for an interferogram formed from acquisitions with the same azimuth misregistration relative to a stack master, the impact of NESD cannot be identified because both the master and slave burst images experience the identical phase ramps, which cancel out in the interferogram.

Azimuth misregistration relates to orbital error in the azimuth direction $\left(O^{\epsilon}\right)$ by $O^{\epsilon}=x \tau v_{s}$. Since $x$ is relative to a stack master, $O^{\epsilon}$ is also relative to the orbit of the stack master. The 1-sigma uncertainty of Sentinel-1 precise orbits in three dimensions (i.e., along-track, cross-track, and radial directions) is approximately $5 \mathrm{~cm} \mathrm{[27].} \mathrm{Assuming} \mathrm{the} \mathrm{same}$ uncertainty in all three directions and ignoring the covariance among the three components, the orbital uncertainty in the azimuth direction is thus approximately $2.9 \mathrm{~cm}$.

The standard deviation of the azimuth misregistration time series of $1.1 \mathrm{e}-3$ to $2 \mathrm{e}-3$ azimuth cell is equivalent to 1.6 to $2.8 \mathrm{~cm}$ orbital uncertainty in the azimuth direction, respectively (assuming $\tau=2.056 \mathrm{e}-3 \mathrm{~s}$ and $v_{s}=7000 \mathrm{~m} / \mathrm{s}$ ). The

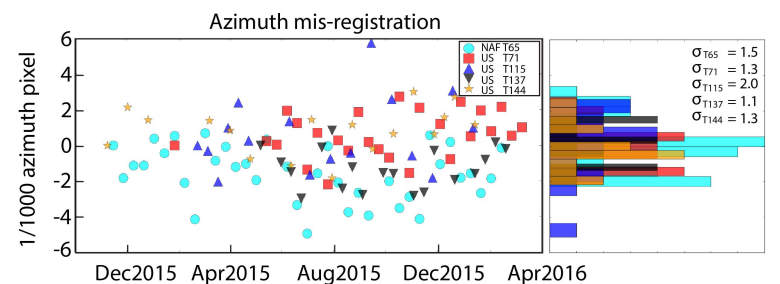

Fig. 8. Azimuth misregistration for different tracks. (Left) Time series of estimated azimuth misregistration for different tracks relative to their first acquisition. (Right) Histogram of the misregistration for different tracks. Each histogram is shifted to its average.

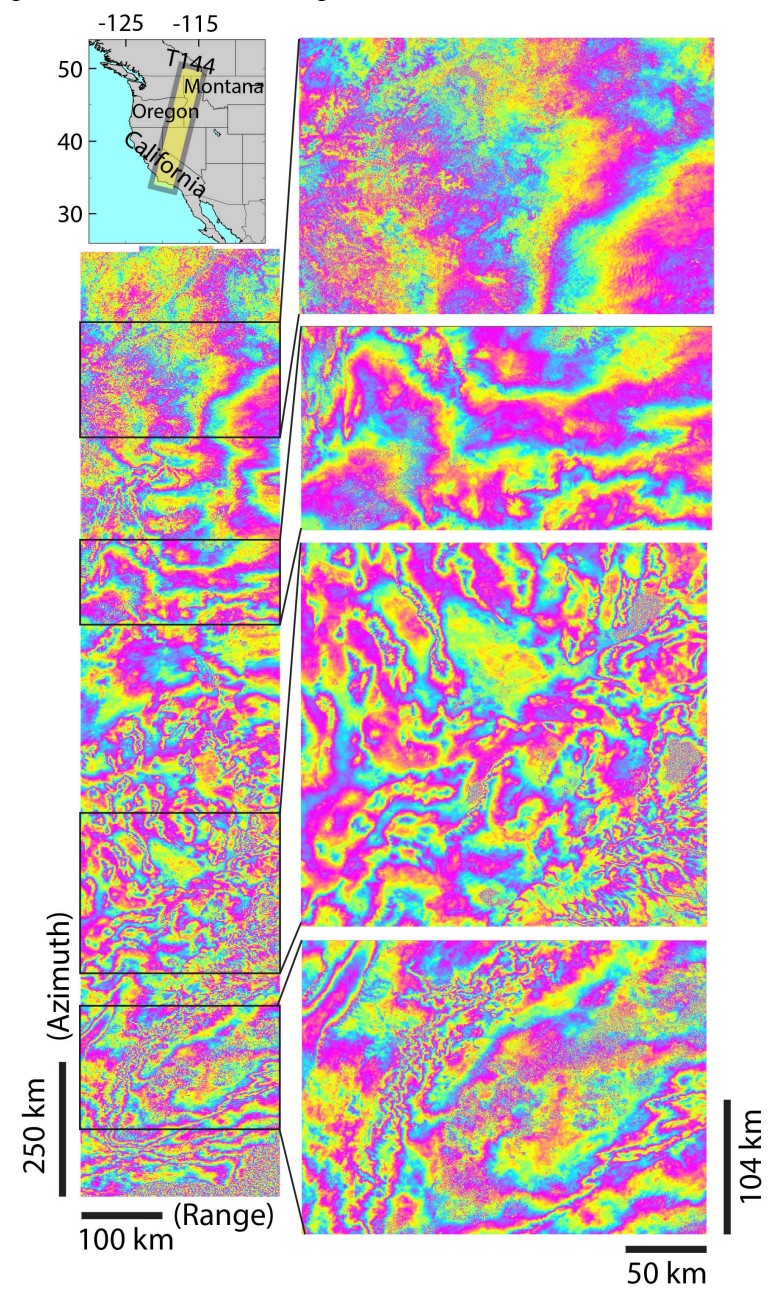

Fig. 9. Large swath Sentinel-1A TOPS interferogram across U.S. from Mexico border to Canada (track 144, 20150706-20150730). The interferometric phase is smooth at the burst boundaries after a precise coregistration using geometry and a constant azimuth misregistration of 0.002 pixels from ESD. Range extension: $250 \mathrm{~km}$. Azimuth extension: $1750 \mathrm{~km}$.

equivalent orbital uncertainty fall within one sigma uncertainty of the precise orbits in the azimuth direction implying that orbital uncertainty is most likely responsible for the constant azimuth misregistration in Sentinel-1 TOPS images. Nevertheless, the misregistration may be partly due to the effect of solid earth tides [28], which have not been compensated for, but could be accounted for with relative ease.

\section{B. Spatial Variation of the Azimuth Misregistration}

The variation of the azimuth misregistration is negligible for Sentinel-1A TOPS along five tracks (tracks 115, 137, 144, and 


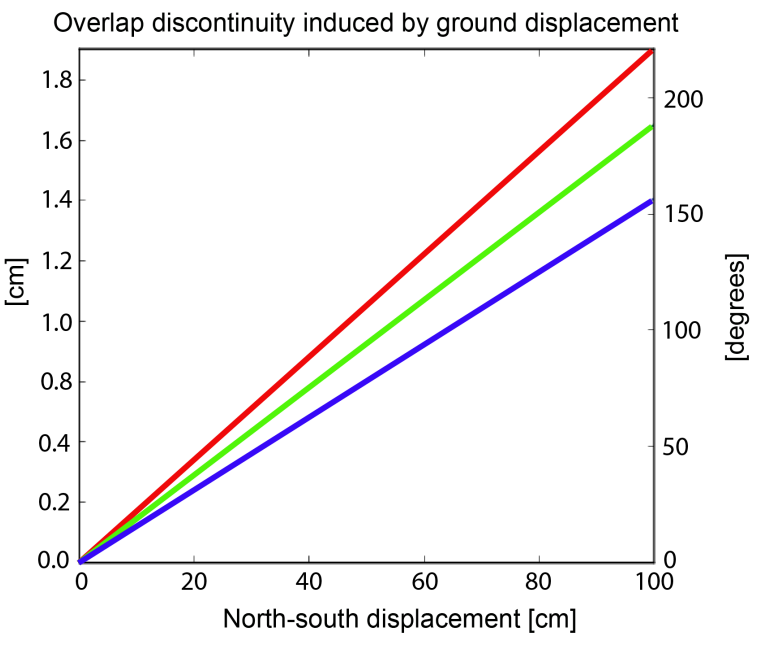

Fig. 10. Expected burst overlap discontinuity in Sentinel-1 TOPS interferograms induced by large $\mathrm{N}-\mathrm{S}$ ground displacement for different incidence angles in near (blue), mid (green), and far (red) ranges.

71 traversing the western U.S. and track 65 traversing Turkey) which contain 10-25 bursts. We find that even for a long multislice interferogram, across the United States from Mexico to Canada (Fig. 9), azimuth misregistration remains constant at 0.002 pixel along this $1750 \mathrm{~km}$ extent. Other studies have also used a constant azimuth offset to remove discontinuities for a long multislice interferogram covering the $2015 \mathrm{Mw} 8.3$ Illapel earthquake [29], [30]. Therefore, recent reports on nonconstant azimuth offsets for the Illapel earthquake by [8] and [31] are more likely due to inconsistent geometry in their processing software rather than actual linear azimuth offsets in the data.

\section{DISCUSSION}

Spectral diversity approaches for TOPS processing, including the ESD and the NESD approach, face different challenges, which may affect the automation of time-series analysis of TOPS data. In this section, we further discuss several such challenges.

\section{A. Large Ground Displacement in the Azimuth Direction}

Azimuth beam steering in TOPS acquisitions leads to a variable squinted line of sight (LOS) direction. The three components of ground displacement in east, north, and vertical directions $\mathbf{u}=\left[u_{e}, u_{n}, u_{u p}\right]$ project to the TOPS LOS direction, $\mathbf{l}_{\mathbf{s}}=\left[\sin (\vartheta) \cos \left(\alpha^{\prime}\right), \sin (\vartheta) \sin \left(\alpha^{\prime}\right), \cos (\vartheta)\right]$ as $\mathbf{u}_{l o s}=$ $\mathbf{u} . \mathbf{l}_{\mathbf{s}}$, where $u_{l o s}$ is the ground-to-satellite displacement in the LOS direction, $\vartheta$ is the incidence angle, and $\alpha^{\prime}=\alpha+\beta$ with $\alpha$ the heading angle and $\beta$ the burst steering angle, which varies between $-0.7^{\circ}$ and $0.7^{\circ}$ across a Sentinel-1 TOPS burst in the azimuth direction. At the overlap of two consecutive burst interferograms, the ground displacement projects to different LOS directions, resulting in discontinuities in the overlap. This effect is not expected for vertical displacement and is maximum for the north-south ground displacement. Fig. 10 shows the expected discontinuity at the burst overlap of Sentinel-1 TOPS interferograms as a function of north-south ground displacement. A relatively large ground displacement of $50 \mathrm{~cm}$ results in $7-\mathrm{mm}$ burst discontinuity equivalent to $94^{\circ}$ phase jump.

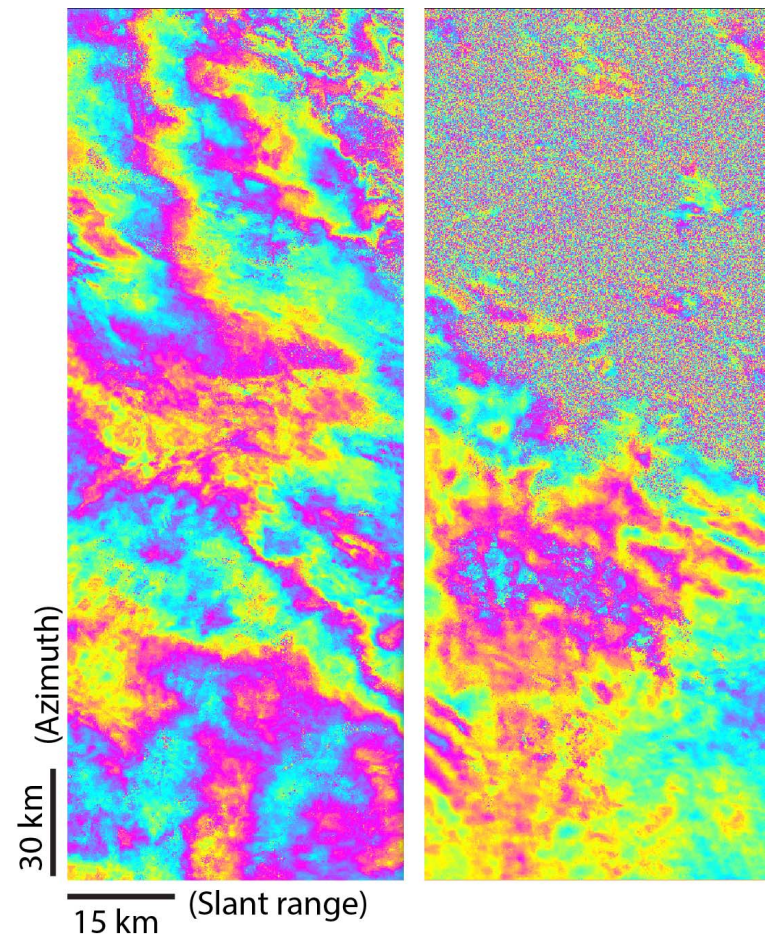

Fig. 11. Two Sentinel-1A TOPS interferograms (track 64, Turkey) formed from (left) summer acquisitions (20150713-20150725) and (right) winter acquisitions (20151122-20151204). The winter pair shows lower coherence (due to snowfall) compared with the summer pair.

Such a large phase difference can significantly bias the estimated misregistration from the spectral diversity. In cases where a model of the ground displacement is available, one possible solution would be to project the displacement to the LOS and remove from the interferograms before estimating the azimuth misregistration. If estimating a constant azimuth misregistration is sufficient, an alternative approach excludes the burst overlaps in the regions of large ground displacement for the ESD and the NESD analysis.

\section{B. Abrupt Coherence Loss}

The main assumption in the NESD approach is that the short temporal baseline interferograms are more coherent compared with the long temporal baseline pairs. Although this assumption is generally valid, it may be violated in some cases when the scattering properties of the scene abruptly change, causing phase decorrelation in small temporal baseline interferograms. Fig. 11 shows that a 12 days winter Sentinel1A TOPS interferogram across the north Anatolian Fault in Turkey is less coherent compared with a 12 days summer interferogram from the same region. The low coherence in the winter interferograms can lead to inaccurate estimation of the azimuth misregistration. A possible solution uses the overlap interferograms from the coherent parts of the interferograms to estimate the misregistration. An alternative is to use longer temporal baseline overlap interferograms for the NESD analysis. Biased single pair misregistration from decorrelated pairs can propagate in the inversion to estimate the misregistration time series in the NESD approach.

To demonstrate the impact of an outlier in the differential misregistration on the misregistration time series, we design a 

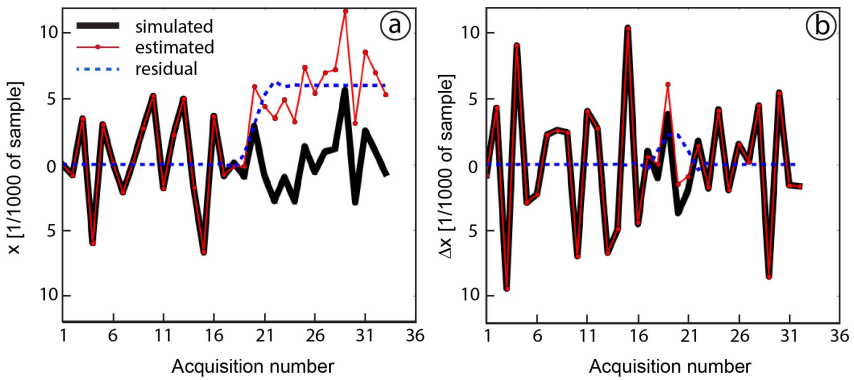

Fig. 12. Simulation showing the impact of outliers on (a) misregistration time series and (b) differential misregistration. In (a), solid black line represents the true misregistration time series, red solid line shows the estimated misregistration time series from a sequential network $(n=2)$, with 0.005 pixel bias added to all observations associated with 20th acquisition, and blue dashed line shows the difference of the estimated and true misregistration. (b) Differential of (a).

simulation. For this simulation, we assume a true misregistration time series with Gaussian distribution (standard deviation of 0.003 pixel) over one year of acquisitions with 12 days interval. From the simulated time series, we form a sequential network of misregistration [with $n=2$ similar to Fig. 4(b)]. We add a bias of 0.005 sample to all simulated differential misregistration associated with 20th acquisition and then we use NESD to estimate the misregistration time series. The acquisitions can be evaluated in three groups; the first group includes those acquisitions before the 20th acquisition and not connected to the 20th acquisition or to any acquisition directly connected to the 20th acquisition (i.e., acquisitions 1 to 15), the second group includes the acquisitions directly connected to the 20th acquisition or connected to one of the acquisitions that is directly connected to the 20th acquisition (i.e., acquisitions 16 to 24), and the third group is similar to the first group but for the acquisitions after the 20th acquisition (i.e., acquisitions 25 to the end).

For a time series relative to the first acquisition, the simulation in Fig. 12 shows that the outlier does not affect the misregistration time series of the first group. The misregistration is biased for the second group. The estimated misregistration time series is offset by a constant for the acquisitions of the third group. The relative differential misregistration among the acquisitions of the first and the third group is not affected by the outlier.

\section{Large Azimuth Misregistration}

The azimuth misregistration can be estimated unambiguously within $\pm\left(1 /\left(2 \Delta f_{\text {ovl }}\right)\right)$ corresponding to \pm 71 to $\pm 85 \mathrm{~cm}$ for Sentinel-1 TOPS subswaths [21]. Given the orbital uncertainty of the Sentinel-1 orbits of $2.9 \mathrm{~cm}$ in along-track direction, the misregistration due to orbital uncertainty falls within the ambiguity band. The estimated azimuth misregistration for different Sentinel-1A tracks in this paper does not exceed $14 \mathrm{~cm}$, which is smaller than the maximum unambiguous azimuth misregistration. However, in the case of large misregistration (larger than the ambiguity band), one can first adjust the geometric coregistration with less accurate coregistration approaches such as the cross-correlation approach and then refine the offsets with more accurate spectral diversity approaches [18].

\section{Nonstationary Azimuth Misregistration}

An assumption inherent in the ESD and NESD approaches is that the azimuth misregistration is constant or only smoothly variable in the azimuth direction, i.e., the azimuth misregistration is stationary and can be modeled with a lower order polynomial in the azimuth direction. This assumption is generally valid when orbital uncertainty or SAR timing error is the source of the azimuth misregistration. The assumption can be violated when azimuth misregistration varies in range direction or when the variation in the azimuth direction is irregular, i.e., azimuth misregistration is nonstationary.

One source of the nonstationary azimuth misregistration is the deviation of the processing geometry (e.g., during SAR image formation) from the real TOPS acquisition geometry. In particular, most $\mathrm{SAR}$ processing schemes assume a constant effective velocity, $v_{\text {eff }}$, to process the SAR data. However, in reality, for typical spaceborne SAR platforms (e.g., TerraSAR$X$ and Sentinel-1), effective velocity varies linearly with topographic height and as a quadratic form with respect to the squint angle [20]. Approximations, such as considering an ellipsoid surface or an average topography height instead of an exact topography or ignoring a squint angle variation (e.g., induced by TOPS geometry) during the SAR image focusing, deviate the effective velocity from the actual effective velocity, causing phase and azimuth positioning errors. The latter translates to azimuth misregistration between TOPS images in the InSAR products. Although the errors may be significant in individual SAR images, they may mostly cancel out in interferometric products formed from similar TOPS acquisitions, because both the master and slave acquisitions contain the same errors [20]. However, inconsistency between the master and slave TOPS images (e.g., different assumptions on reference topography height used for focusing the two images) can expose the errors in the interferometric applications.

Another source of the nonstationary azimuth misregistration is the inhomogeneity in ionospheric propagation delay. Inhomogeneous ionosphere between SAR acquisitions causes variable azimuth misregistration, which cannot be corrected with a constant offset assumption.

Fig. 13 shows the third swath of two interferograms from Track 149 in Northern Chile, and their associated azimuth offsets at the burst overlaps obtained with ESD. The first interferogram does not show any phase discontinuity at the burst overlaps; its azimuth offset seems constant across the SAR scene. The second interferogram shows phase discontinuity at burst overlaps; their azimuth offsets indicate that the misregistration varies in space and cannot be corrected with a constant offset. We hypothesize that the misregistration in the second interferogram is due to the effect of the ionosphere.

\section{E. Refining the Offsets of Stack of SAR Images Versus the Offsets of Individual Pairs}

The theoretical uncertainty of the misregistration time series obtained with NESD (Fig. 4) indicates the temporal accumulation of uncertainty with respect to the stack master. However, 

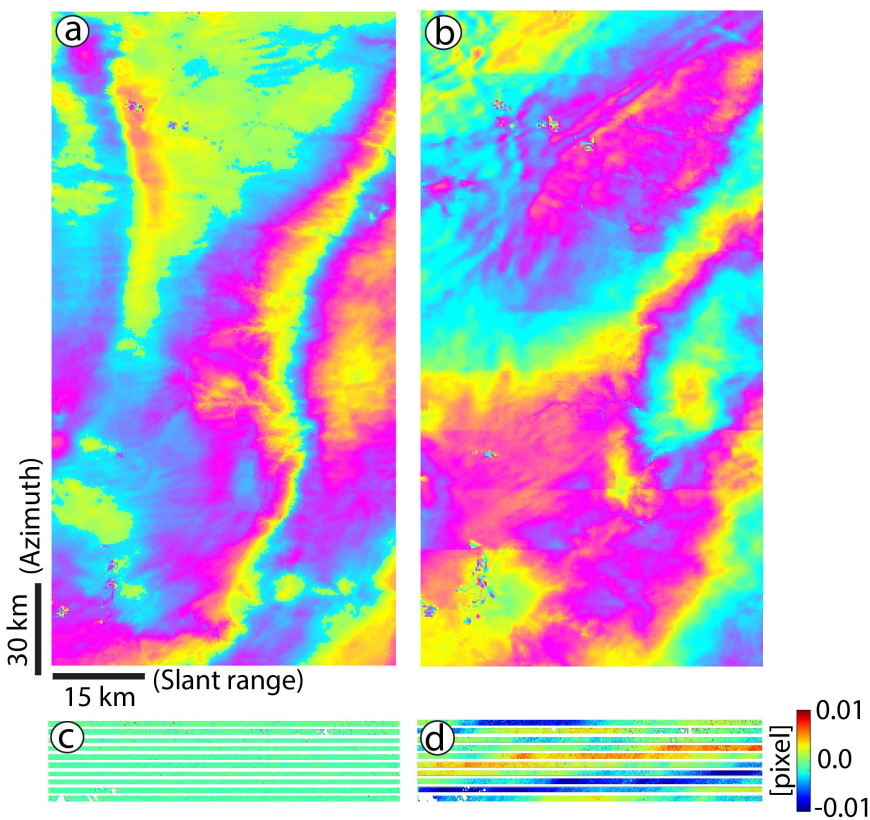

Fig. 13. Example interferograms from track 149 over Northern Chile with constant and variable azimuth misregistration. (a) 20150917-20151011 pair, (b) 20151128-20151222 pair, (c) azimuth misregistration at the overlap regions of (a), and (d) azimuth misregistration at the overlap regions of (b). The offsets are constant in (c) and variable in (d). The phase discontinuities in (b) are due to the variable azimuth misregistration, which is most likely associated with the ionospheric inhomogeneity.

due to two primary reasons, NESD should be used to refine the offsets of a stack of TOPS images with respect to a stack master instead of refining the relative offsets of TOPS image pairs for each interferogram independently.

First, refining the offsets of the stack of SAR images using a misregistration time series maintains geometrical consistency within the stack, while individual pair refinement can lead to inconsistencies. Consistency among interferograms can be evaluated by computing a phase close circuit [32]. Even if the individual pair misregistration is consistent, their uncertainty propagates to InSAR time-series products similar to the misregistration time series obtained with NESD. Therefore, a stack of interferograms, in which the offsets between pairs are refined independently for each pair, at best, results in the same uncertainty as a stack corrected with NESD, but with the risk of inconsistency.

Second, the impact of the misregistration on the InSAR displacement time series, obtained with InSAR time-series analysis techniques, such as small-baseline or permanent scatterer techniques [33], [34], is proportional to the misregistration time series. This is similar to the impact of geometrical residuals such as the effect of DEM error on InSAR time series, in which the impact is proportional to the baseline time series of the SAR acquisitions and not on the baseline between the individual pairs [35]. Accordingly, even if the impact of misregistration cannot be identified in an individual interferogram formed from two SAR acquisitions with identical misregistration relative to a stack master, the misregistration of the two acquisition contributes to the time series with respect to the stack master, and therefore, the offsets of both acquisitions must be refined relative to the stack master.

\section{CONCLUSION}

We developed a workflow for estimating the time series of azimuth misregistration among TOPS acquisitions using an NESD. Misregistration time series is required for precise coregistration of a stack of TOPS acquisitions to a given coordinate system. Time series of misregistration from five stacks of Sentinel-1 TOPS acquisitions, obtained with NESD, indicates maximum relative azimuth offsets of less than 0.01 azimuth cell and standard deviations of $1.1 \mathrm{e}-3$ to $2 \mathrm{e}-3$ azimuth cell, equivalent to orbital uncertainty of 1.6 to $2.8 \mathrm{~cm}$, respectively, falling within the 1-sigma orbital uncertainty of the Sentinel-1 precise orbits.

The NESD workflow can be potentially operationalized to provide and update the time series of the misregistration for each Sentinel-1 track. Evaluating the uncertainty of misregistration time series obtained with NESD based on the misregistration uncertainty of the individual pairs indicates that the uncertainty of the misregistration time series increase with time. However, for constant azimuth misregistration obtained from averaging the offsets from many coherent overlap pixels, uncertainties better than 0.001 azimuth cell are achievable even for long time series (e.g., ten years). We also evaluated different challenges for operationalizing NESD, including the impact of large ground displacement in the azimuth direction, and the effect of decorrelation and outliers on the estimated misregistration time series. ESD and NESD can mitigate the phase discontinuities due to constant (or smoothly varying in the azimuth direction) azimuth misregistration. However, adjusting spatially variable azimuth misregistration (e.g., caused by inhomogeneous ionosphere) requires further investigations.

\section{ACKNOWLEDGMENT}

The Sentinel-1 data were provided by ESA/Copernicus. Part of the research was carried out at the Jet Propulsion Laboratory, California Institute of Technology, under a contract with the National Aeronautics and Space Administration. The authors would like to thank N. Miranda from the European Space Agency for the helpful discussions on focusing terrain observation by progressive scan data.

\section{REFERENCES}

[1] F. De Zan and A. Monti Guarnieri, "TOPSAR: Terrain observation by progressive scans," IEEE Trans. Geosci. Remote Sens., vol. 44, no. 9, pp. 2352-2360, Sep. 2006.

[2] R. Torres et al., "GMES Sentinel-1 mission," Remote Sens. Environ., vol. 120, pp. 9-24, May 2012.

[3] A. Meta, J. Mittermayer, P. Prats, R. Scheiber, and U. Steinbrecher, "TOPS imaging with TerraSAR-X: Mode design and performance analysis,' IEEE Trans. Geosci. Remote Sens., vol. 48, no. 2, pp. 759-769, Feb. 2010.

[4] P. Prats-Iraola, R. Scheiber, L. Marotti, S. Wollstadt, and A. Reigber, "TOPS interferometry with TerraSAR-X," IEEE Trans. Geosci. Remote Sens., vol. 50, no. 8, pp. 3179-3188, Aug. 2012.

[5] M. Nannini, P. Prats-Iraola, F. De Zan, and D. Geudtner, "TOPS time series performance assessment with TerraSAR-X data," IEEE J. Sel. Topics Appl. Earth Observ. Remote Sens., vol. 9, no. 8, pp. 3832-3848, Aug. 2016.

[6] J. R. Elliott et al., "Himalayan megathrust geometry and relation to topography revealed by the Gorkha earthquake," Nature Geosci., vol. 9, pp. 174-180, Jan. 2016. 
[7] F. Casu, C. De Luca, I. Zinno, and M. Manunta. (Apr. 2016). Sentinel-1 T163 Co-Seismic Interferogram of Kumamoto EQ. [Online]. Available: http://dx.doi.org/10.5281/zenodo.50209

[8] R. Grandin, E. Klein, M. Métois, and C. Vigny, "Three-dimensional displacement field of the $2015 \mathrm{Mw} 8.3$ Illapel earthquake (Chile) from across- and along-track Sentinel-1 TOPS interferometry," Geophys. Res. Lett., vol. 43, no. 6, pp. 2552-2561, 2016.

[9] H. Fattahi. (2016). Sentinel-1A Interferogram for Tajikistan Earthquake. [Online]. Available: https://winsar.unavco.org/portal/insar/36/

[10] P. González et al., "The 2014-2015 eruption of Fogo volcano: Geodetic modeling of Sentinel-1 TOPS interferometry," Geophys. Res. Lett., vol. 42, no. 21, pp. 9239-9246, 2015.

[11] T. Nagler, H. Rott, M. Hetzenecker, J. Wuite, and P. Potin, "The Sentinel-1 mission: New opportunities for ice sheet observations," Remote Sens., vol. 7, no. 7, pp. 9371-9389, Jul. 2015. [Online]. Available: http://www.mdpi.com/2072-4292/7/7/9371/

[12] R. Bamler, "Interferometric stereo radargrammetry: Absolute height determination from ERS-ENVISAT interferograms," in Proc. Int. Geosci. Remote Sens. Symp. (IGARSS), vol. 2. 2000, pp. 742-745.

[13] R. Bamler and M. Eineder, "Split band interferometry versus absolute ranging with wideband SAR systems," in Proc. IGARSS, 2004, pp. $980-984$.

[14] R. Bamler and M. Eineder, "Accuracy of differential shift estimation by correlation and split-bandwidth interferometry for wideband and delta-k SAR systems," IEEE Geosci. Remote Sens. Lett., vol. 2, no. 2 , pp. 151-155, Apr. 2005.

[15] E. Sansosti, P. Berardino, M. Manunta, F. Serafino, and G. Fornaro, "Geometrical SAR image registration," IEEE Trans. Geosci. Remote Sens., vol. 44, no. 10, pp. 2861-2870, Oct. 2006.

[16] F. De Zan, P. Prats-Iraola, R. Scheiber, and A. Rucci, "Interferometry with TOPS: Coregistration and azimuth shifts," in Proc. EUSAR, 2014, pp. 949-952.

[17] A. Monti Guarnieri, S. Mancon, and S. Tebaldini, "Sentinel-1 precise orbit calibration and validation," in Proc. FRINGE Workshop, Frascati, Italy, 2015.

[18] R. Scheiber, M. Jäger, P. Prats-Iraola, F. De Zan, and D. Geudtner, "Speckle tracking and interferometric processing of TerraSAR-X TOPS data for mapping nonstationary scenarios," IEEE J. Sel. Topics Appl. Earth Observ. Remote Sens., vol. 8, no. 4, pp. 1709-1720, Apr. 2015.

[19] R. Scheiber and A. Moreira, "Coregistration of interferometric SAR images using spectral diversity," IEEE Trans. Geosci. Remote Sens., vol. 38, no. 5, pp. 2179-2191, Sep. 2000.

[20] M. Rodriguez-Cassola et al., "Doppler-related distortions in TOPS SAR images," IEEE Trans. Geosci. Remote Sens., vol. 53, no. 1, pp. 25-35, Jan. 2015.

[21] N. Yagüe-Martínez et al., "Interferometric processing of Sentinel-1 TOPS data," IEEE Trans. Geosci. Remote Sens., vol. 54, no. 4 , pp. 2220-2234, Apr. 2016.

[22] N. Yagüe-Martínez, P. Prats-Iraola, and F. De Zan, "Coregistration of interferometric stacks of Sentinel-1A TOPS data," in Proc. 11th Eur. Conf. Synth. Aperture Radar (EUSAR), 2016, pp. 884-889.

[23] K. Tomiyasu, "Conceptual performance of a satellite borne, wide swath synthetic aperture radar," IEEE Trans. Geosci. Remote Sens., vol. GE-19, no. 2, pp. 108-116, Mar. 1981.

[24] R. K. Moore, J. P. Claassen, and Y. H. Lin, "Scanning spaceborne synthetic aperture radar with integrated radiometer," IEEE Trans. Aerosp. Electron. Syst., vol. AES-17, no. 3, pp. 410-421, May 1981.

[25] R. Bamler, "Optimum look weighting for burst-mode and ScanSAR processing," IEEE Trans. Geosci. Remote Sens., vol. 33, no. 3, pp. 722-725, May 1995.

[26] M. Shimada, "A new method for correcting ScanSAR scalloping using forests and inter-SCAN banding employing dynamic filtering," IEEE Trans. Geosci. Remote Sens., vol. 47, no. 12, pp. 3933-3942, Dec. 2009.

[27] C. Fernández, Sentinels POD Team, "Sentinels POD service file format specifications," Eur. Space Agency, Paris, France, Tech. Rep. GMESGSEG-EOPG-FS-10-0075, 2011.

[28] M. Eineder, C. Minet, P. Steigenberger, X. Cong, and T. Fritz, "Imaging geodesy-Toward centimeter-level ranging accuracy with TerraSAR-X," IEEE Trans. Geosci. Remote Sens., vol. 49, no. 2, pp. 661-671, Feb. 2011.

[29] G. Solaro et al., "Coseismic fault model of Mw 8.32015 Illapel earthquake (Chile) retrieved from multi-orbit Sentinel 1-A DInSAR measurements," Remote Sens., vol. 8, no. 4, p. 323, Apr. 2016. [Online]. Available: http://www.mdpi.com/2072-4292/8/4/323

[30] D. Melgar et al., "Slip segmentation and slow rupture to the trench during the 2015, $M_{w} 8.3$ Illapel, Chile earthquake," Geophys. Res. Lett., vol. 43 , no. 3, pp. 961-966, 2016.
[31] B. Xu et al., "Continent-wide 2-D co-seismic deformation of the $2015 \mathrm{Mw} 8.3$ Illapel, Chile earthquake derived from Sentinel-1A data: Correction of azimuth co-registration error," Remote Sens., vol. 8, no. 5, p. 376, May 2016. [Online]. Available: http://www.mdpi.com/2072$4292 / 8 / 5 / 376$

[32] P. S. Agram and M. Simons, "A noise model for InSAR time series," J. Geophys. Res., Solid Earth, vol. 120, no. 4, pp. 2752-2771, 2015.

[33] P. Berardino, G. Fornaro, R. Lanari, and E. Sansosti, "A new algorithm for surface deformation monitoring based on small baseline differential SAR interferograms," IEEE Trans. Geosci. Remote Sens., vol. 40, no. 11, pp. 2375-2383, Nov. 2002.

[34] A. Ferretti, A. Fumagalli, F. Novali, C. Prati, F. Rocca, and A. Rucci, "A new algorithm for processing interferometric data-stacks: SqueeSAR," IEEE Trans. Geosci. Remote Sens., vol. 49, no. 9, pp. 3460-3470, Sep. 2011.

[35] H. Fattahi and F. Amelung, "DEM error correction in InSAR time series," IEEE Trans. Geosci. Remote Sens., vol. 51, no. 7, pp. 4249-4259, Jul. 2013.

[36] A. Monti Guarnieri and S. Tebaldini, "Hybrid Cramér-Rao bounds for crustal displacement field estimators in SAR interferometry," IEEE Signal Process. Lett., vol. 14, no. 12, pp. 1012-1015, Dec. 2007.

[37] A. Monti Guarnieri and S. Tebaldini, "On the exploitation of target statistics for SAR interferometry applications," IEEE Trans. Geosci. Remote Sens., vol. 46, no. 11, pp. 3436-3443, Nov. 2008.

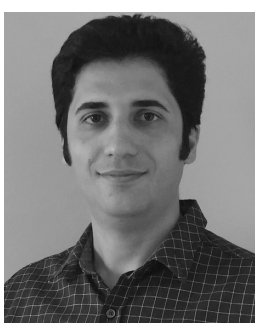

Heresh Fattahi (M'12) received the M.S. degree in remote sensing engineering from the K.N. Toosi University of Technology, Tehran, Iran, in 2007, and the Ph.D. degree in geosciences from the University of Miami, Coral Gables, FL, USA, in 2015.

Since 2015, he has been a Postdoctoral Scholar with the California Institute of Technology, Pasadena, CA, USA. His current research interests include interferometric synthetic aperture radar (InSAR) time-series analysis, uncertainty of the InSAR measurements of ground displacement, and algorithm development for TOPS time-series analysis and for correction of ionospheric propagation delay in synthetic aperture radar data.

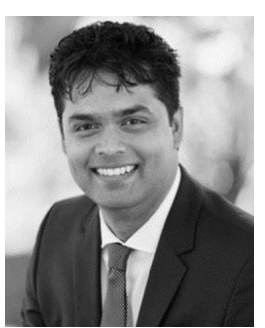

Piyush Agram (M'10) received the B.Tech. degree in electrical engineering from IIT Madras, Chennai, India, in 2004, and the Ph.D. degree in electrical engineering from Stanford University, Stanford, CA, USA, in 2010

$\mathrm{He}$ was with the California Institute of Technology's Seismological Laboratory, Keck Institute of Space Studies, Pasadena, CA, USA, as a Postdoctoral Scholar until 2013. $\mathrm{He}$ then joined the Radar Algorithms and Processing Group, Jet Propulsion Laboratory, Pasadena. His current research interests include algorithm development for synthetic aperture radar focusing, radar interferometry for deformation time-series applications, and geospatial big data analysis.

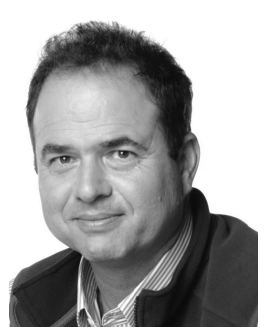

Mark Simons received the B.Sc. degree in geophysics and space physics from the University of California at Los Angeles, Los Angeles, CA, USA, in 1989, and the Ph.D. degree in geophysics from the Massachusetts Institute of Technology, Cambridge, MA, USA, in 1996.

Since 1996, he has been with the California Institute of Technology, Pasadena CA, USA, where he is currently a Professor of geophysics in the Seismological Laboratory within the Division of Geological and Planetary Sciences. His current research interests include studying processes that deform the solid earth including those associated with the seismic cycle, migration of magma and water in the subsurface, tides, and glacial rebound; tectonics and the relationship between short and long time scale processes; glaciology, particularly basal mechanics and ice rheology; tools and applications using space geodesy, particularly Global Navigation Satellite System and synthetic aperture radar; Bayesian methods for large geophysical inverse problems; and application of space geodesy for monitoring and rapid response to natural disasters. 